\title{
Multifunctional Polymeric Carriers for Gene and Drug Delivery
}

\author{
Tatiana Bronich
}

Received: 31 August 2010 / Accepted: 31 August 2010 / Published online: 16 September 2010

(C) Springer Science+Business Media, LLG 2010

The need for the discovery and development of innovative technologies to improve the delivery of therapeutic and diagnostic agents is broadly acknowledged. Such technologies are expected to minimize drug degradation upon administration and maximize the therapeutic efficacy of the drug by delivering it to the site of the action in desired quantities while limiting the adverse side effects. During the last few decades, the field of biomaterials for delivery of low molecular drugs, proteins, and nucleic acids has seen exponential growth. It is now well accepted that modern drug carriers need to be multicomponent to be able to overcome many physical and biochemical barriers in the body before a drug molecule can reach a target site. The inherent multifunctionality of polymers (synthetic and natural) offers a unique advantage for the design of such systems. The drugs can be physically mixed or covalently conjugated with polymers to improve their solubility and stability upon administration and facilitate prolonged blood circulation. They can be designed to be stimuli-responsive for releasing therapeutic loads in a site- and time-specific manner. Multiple targeting moieties can be attached to the macromolecular carrier to direct drugs to a certain organ, tissue, cell, or cellular compartment. Recently, polymers were also used to design integrated theranostic systems that combine both therapeutic and diagnostic functionalities and enable image-guided, controlled, and minimally invasive therapies for cancer and other devastating human diseases.

T. Bronich $(\bowtie)$

Center for Drug Delivery and Nanomedicine

University of Nebraska Medical Center

Omaha, Nebraska, USA

e-mail: tbronich@unmc.edu
Moreover, there is growing evidence that some synthetic polymers can display biological response-modifying activity and can influence the molecular mechanism of action of a drug. This property can be further exploited to improve delivery and drug or gene performance.

Numerous polymer-based drug and gene delivery carriers, including polymer-drug conjugates, polymer micelles, polymer-coated liposomes, nanogels, and nanoparticles, are being developed. The remarkable and rapid progress in the area of multifunctional polymer therapeutics justifies the appearance of this special section that covers major topics in this fast-growing field. Translation of nucleic acid-based therapy to clinical studies requires significant advances in the delivery systems that will mitigate the undesirable biological properties of the nonviral gene carriers, such as toxicity, instability in the bloodstream, poor cellular internalization, and insufficient intracellular trafficking. Dr. Glen Kwon and colleagues describe how the reversible PEGgylation of DNA/polycation complexes can provide for high colloidal stability of the gene carrier without compromising transfection efficiency. Dr. Karel Ulbrich reminds us that polymers can also be effectively used for surface modification of adenoviral gene delivery vector. The authors developed an elegant technique for controlled conjugation of the targeting moieties to the vector and demonstrated the redirection of the natural viral tropism to receptor-specific adenovirus gene transfer. Dr. Vinod Labhasetwar's group used hydrophilic polymer, poly(ethylene glycol), for surface modification of hydrophobic supermagnetic iron oxide nanoparticles. They demonstrated the feasibility of developing multifunctional nanoparticles with specific, cancer-targeting MRI detection and sustained release of the drug for image-guided cancer therapy. 
The concept of polymer-drug conjugation was proposed in the 1970s and has unquestionably become a practical tool to the pharmaceutical scientists. In this issue, the report by Dr. Tamara Minko and colleagues aims to elucidate in more detail the therapeutic potential of polymer-peptidedrug conjugates based on poly(ethylene glycol) and containing multiple copies of anticancer drug (camptothecin) together with a tumor-targeting moiety (a synthetic analog of luteinizing hormone-releasing hormone) and a suppressor of cellular antiapoptotic defense (BH3 peptide). They clearly demonstrate that the antitumor activity of peptidedrug conjugates increased with an increase in number of copies of each active component conjugated to one molecule of the carrier. Dr. Hamidreza Ghandehari's group continues to explore three-dimensional and flexible architecture of dendrimers in design of delivery systems. They have previously shown that complexes of PAMAM dendrimers and camptothecin analogs improve the transepithelial transport and cellular uptake of the drug. In this issue, they report on covalent conjugates of such systems so that non-specific release in the bloodstream is reduced and release is facilitated at the target site. Studies by Dr. Julia Lubimova and coauthors are focused on poly $(\beta$-L-malic acid), a biodegradable, non-toxic, and non-immunogenic polymer, as a platform for conjugation of temozolomide, a potent chemotherapeutic agent for treatment of malignant glioma. Previously, they have successfully delivered antisense oligonucleotides specifically to brain tumor using a similar strategy.

Three reports in this issue are focused on polymer micelles, another promising modality of macromolecular drug delivery systems. Dr. Younsoo Bae and coauthor explore a concept of tunable drug release using polymer micelles. They emphasize the importance of careful tailoring of polymer-drug cleavable linkers for the design of macromolecular prodrugs that are able to display sitespecific drug release with a predetermined profile. Dr. Christine Allen and colleagues utilize the block copolymer micelles labeled with indium-111 and decorated by EGF targeting moieties to elucidate the peculiarities of pharmacokinetics, biodistribution, and intratumoral transport of polymer micelles. They conclude that an adequate passive targeting of macromolecular carriers is required in order to achieve effective active targeting. The authors also highlight that tumor physiology, low levels of cell uptake, and continuous exocytosis of internalized polymer micelles remain formidable challenges in achieving a therapeutically relevant active targeting effect in vivo. Dr. Dong Wang's group employs micellar carriers based on Pluronic copolymers terminated with mineral binding moiety in order to enhance the delivery of the antimicrobial agent, triclosan, to the tooth surface. The results showed that targeted micellar formulations of triclosan inhibited the formation of plaque biofilms and effectively reduced the viability of preformed biofilms. Consequently, the proposed micellar antimicrobial agent has the potential to be effective in the prevention and treatment of dental caries.

We are extremely grateful to all authors for their valuable contributions enabling the appearance of this special theme section. The research directions outlined in these reports show great promise in improving safe and effective delivery of bioactive agents to the site of action in therapeutically relevant quantities. We believe that these reports will be of interest to a very broad group of readers of Pharmaceutical Research.

\section{INTERVIEW WITH DR. TATIANA K. BRONICH}

\section{What holds the key to your success as a pharmaceutical scientist?}

I do not know to what extent I am really successful, but one of its measures is the amount of joy you derive from what you have done. Besides that, a measure of our success is the success of our colleagues, collaborators, and students.

\section{What do you consider to be your key research accomplishments?}

I would consider myself lucky if the knowledge that was gained in our research could be translated into the future clinical practice and be beneficial in fighting human disease.

\section{What was the turning point in your career?}

I always have been interested in understanding how various polymer molecules recognize and interact with each other and form supramolecular structures. When I joined the Department of Pharmaceutical Sciences at UNMC, it became exciting to include the use of the supramolecular assembly as a tool to produce polymeric nanostructured materials for application in drug delivery.

\section{Which individuals have most influenced your research career?}

I am very fortunate to have had several in my life, including my mother, a chemistry professor, and my mentor, Professor Victor Kabanov at Moscow State University. I have always admired their dedication to science and their unconditional love for and belief in the people around them. I credit Dr. Alexander Kabanov, an exceptional scientist, great collaborator, and friend, for making a difference in my scientific career and introducing me to the field of pharmaceutical sciences. 
Pharmaceutical scientists are faced with the dilemma of having to publish in biomedical or basic science journals. Does this mean cutting-edge science will not likely be featured in the pharmaceutical research?

I think that the special section on multifunctional polymers in Pharmaceutical Research provides an answer to this question: the included papers are related to the science frontiers in this field.

Where is the field of biomaterials for nucleic acid and drug delivery going, and how do the articles in this theme section fill the gap?

During the last few decades, the field of biomaterials for delivery of low molecular drugs, proteins, and nucleic acids has seen exponential growth. Tremendous versatility of modern polymer chemistry allows for a steady introduction of new macromolecular carriers with tunable size, shape, and surface properties. A major advantage of such systems - their multifunctionality-continues to be explored to facilitate a controlled delivery of therapeutic and diagnostic agents to specific tissues, cells, and cellular compartments. The articles collected in this theme section represent some of the latest advancements in this research area.

What are the challenges for designing and using novel multifunctional polymers as carrier molecules, and how can they be overcome?

It is already obvious that the effective polymeric carriers need to be multifunctional to be able to cross the existing barriers in the body, avoid recognition by immune cells, and deliver their payload in the optimum dosage range to the site of the disease in a precise manner. Integration of such multiple signaling cues increases the complexity and heterogeneity of the macromolecular carriers and presents challenges for their characterization and subsequent scaleup. It is also clear that relatively small changes in physicochemical characteristics (e.g., polymer composition, particle size, shape, surface chemistry and charge, and targeting functionality) have significant biological implications for the fate of polymer carriers in the body. Further systemic studies of how these structures interact with cells and cellular compartments are needed to improve targeting strategies at both cellular and subcellular levels. Therefore, a careful tailoring of polymer architecture with biological targeting is essential for a successful design of diseaseoriented delivery systems. A multidisciplinary (chemistry, material science and engineering, pharmaceutical sciences, and biology), integrated approach is required to address these challenges and create effective nanomedicines for clinical use.
What is the key to developing successful collaborative relationships, and how can the Center for Drug Delivery and Nanomedicine help?

Trust, mutual respect, and complementary sets of skills are needed to tackle a problem. From this point of view, our Center for Drug Delivery and Nanomedicine, established in 2004 under the leadership of Dr. Alexander Kabanov, serves to combine diverse technical and scientific expertise in biomedical and material science research and engineering available at the University of Nebraska. It integrates faculty members from clinical and basic sciences departments and has already fostered multiple fruitful cross-disciplinary collaborations that support drug delivery research.

\section{What is your philosophy of educating graduate students?}

In my opinion, a research career is an enormous commitment of time. It's not just a job-it's a life. You have to love it, and it will sustain you through the ups and downs. It is very important to maintain focus and always be open-minded.

\section{What are the challenges facing the pharmaceutical sciences?}

Drug delivery still remains a challenge. It includes development of better formulation strategies and the search for targeting moieties for site-specific delivery or delivery to the areas which are not readily accessible. Integration of the recent achievements in nanomedicine research and imaging technologies with progress in our understanding of the molecular basis of the diseases can help to address these problems.

\section{What is the place for collaboration with industry in academia?}

A close collaboration between pharmaceutical scientists in academia and industry is paramount for transforming a scientific concept into a real product.

Dr. Tatiana K. Bronich is a Professor at the College of Pharmacy and Associate Director of the Center for Drug Delivery and Nanomedicine at the University of Nebraska Medical Center. Her educational background includes undergraduate training in Chemistry at Moscow State University, Russia, followed by graduate studies at the Department of Polymer Sciences of the same University. Dr. Bronich's research interests are in the area of self-assembling polymer materials and applications of these materials in medicine. Of special interest is the design and study of novel types of functional materials based on complexes formed between block ionomers andoppositely charged polymers and low-molecular-weight amphiphilic molecules. Her recent work has expanded to include the application of these amphiphilic block copolymers and block ionomer complexes in drug delivery to treat cancer and the development of the polycation-DNA complexes for gene delivery. 